\title{
Signal modeling of an MRI ribbon solenoid coil dedicated to spinal cord injury investigations
}

\author{
Christophe Coillot $^{1}$, Rahima Sidiboulenouar ${ }^{1}$, Eric Nativel $^{2}$, Michel Zanca ${ }^{1,4}$, Eric Alibert ${ }^{1}$, \\ Maida Cardoso $^{1}$, Guillaume Saintmartin ${ }^{1,3}$, Harun Noristani ${ }^{3}$, Nicolas Lonjon ${ }^{3,4}$, Marine Lecorre ${ }^{3,4}$, \\ Florence Perrin $^{3}$, and Christophe Goze-Bac ${ }^{1}$ \\ ${ }^{1}$ Laboratoire Charles Coulomb (L2C-UMR5221), BioNanoNMRI group, University of Montpellier, \\ Place Eugene Bataillon, 34095 Montpellier, France \\ ${ }^{2}$ Institut d'Electronique et des Systèmes (IES-UMR5214), University of Montpellier, \\ Campus Saint-Priest, 34095 Montpellier, France \\ ${ }^{3}$ Institut des Neurosciences de Montpellier (INSERM U1051), University of Montpellier, \\ 34095 Montpellier, France \\ ${ }^{4}$ Nuclear medicine, CMC Gui de Chauliac, University Hospital Montpellier, 34095 Montpellier, France \\ Correspondence to: Christophe Coillot (christophe.coillot@univ-montp2.fr)
}

Received: 23 November 2015 - Revised: 7 March 2016 - Accepted: 23 March 2016 - Published: 6 April 2016

\begin{abstract}
Nuclear magnetic resonance imaging (NMRI) is a powerful tool for biological investigations. Nevertheless, the imaging resolution performance results in the combination of the magnetic field $\left(B_{0}\right)$ and the antenna efficiency. This latter one results in a compromise between the size of the sample, the location of the region of interest and the homogeneity requirement. In the context of spinal cord imaging on mice, a ribbon solenoid coil is used to enhance the efficiency of the MRI experiment. This paper details the calculation of the local magnetization contribution to the induced voltage of MRI coils. The modeling is illustrated on ribbon solenoid antennas used in emitter-receiver mode for the study. The analytical model, which takes into account the emitting mode, the receiving step and the imaging sequence, is compared to the measurement performed on a 9.4 T VARIAN MRI apparatus. The efficiency of the antenna, in terms of signal to noise ratio, is significantly enhanced with respect to a commercial quadrature volumic antenna, given a significant advantage for the study of spinal cord injuries.
\end{abstract}

\section{Context of the study: the spinal cord injuries}

Spinal cord injuries (SCIs) are devastating neuropathologies that affect over 2.5 million patients worldwide, yield major handicaps and represent high costs to our society (from about USD 1 million to up than 4 million per patient, National SCI Statistical Care, Sekhon and Fehlings, 2001). Neurological difficulty depends on the spinal level and lesion severity. Unfortunately, there is no effective treatment for any symptoms associated with SCI. MRI is indeed well-established as the most commonly used imaging approach to diagnose and follow-up spinal cord injury patients. In the context of spinal cord injury studies in animals, MRI allows the localization of the region of the lesion and its evolution in order to understand the fundamental biological mechanisms and the perspective of translation to clinics, to evaluate the effect of therapeutical trials. Even if it is preferentially used for in vivo studies, in vitro imaging of tissue has the advantage of an enhanced resolution because of the acquisition time which is less constrained (Nor et al., 2015).

The aim of the ex vivo MRI study is to deepen the in vivo analysis of altered tissues by means of higher MRI spatial resolution and to evaluate putative correlation with histology. Nevertheless, the imaging resolution performance results from the combination of the magnetic field $\left(B_{0}\right)$, the acquisition time and the antenna efficiency in terms of signal to noise ratio (SNR). By a literal shortcut, MRI experimenters usually define the SNR as the ratio between the 


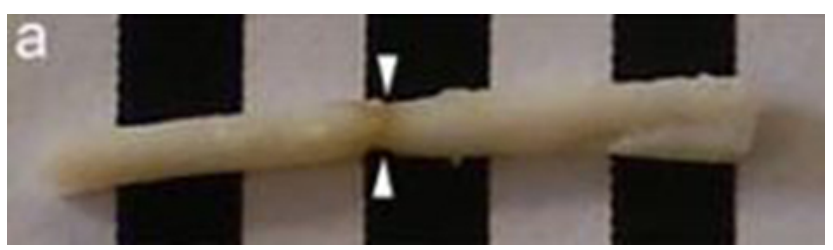

Figure 1. Spinal cord tissue of a CX3CR1 mouse: length is typically about $25-35 \mathrm{~mm}$ and diameter is about $2-3 \mathrm{~mm}$.

mean voxel intensity for a given sample (which is related to the magnetization quantity and to the coil sensitivity (in $V / T)$ ) divided by the voxel intensity in a region outside the sample (which is closely related to the coil plus preamplifier stochastic noise contribution). In the following, we will consider the SNR of MRI experimenters. In order to enhance the imaging performances a dedicated antenna must be designed. The MRI coil design will result in a compromise between the size of the sample, the location of the region of interest and the homogeneity requirement. The requirement homogeneity on the MRI coil is defined (cf. Mispelter et al., 2006 , p. 309) as a $\pm 5 \%$ magnetic field variation. Since we will focus on the final induced voltage, this criterion is excluded. Thus, we define the homogeneity zone as the region where the induced voltage variation remains within $10 \%$ of its maximum. The MRI coils can be used either in emitterreceiver mode or solely in one of the two modes. In case of the separation between emitter and receiver mode an active or passive decoupling is mandatory. Next, an impressive variety of MRI coils have been invented and used (Mispelter et al., 2006): solenoid, saddle coil, loop coil, loop gap, scroll coil and bird cage. The configuration of the sample (its size) and the requirements of the experiment (in terms of SNR and homogeneity) could dictate the choice. In the context of spinal cord tissue (as shown in Fig. 1) the choice of the coil is restricted to the solenoid coil, the scroll coil or the loop gap. The solenoid coil appears to be a relevant choice for simplicity of manufacturing and signal to noise ratio efficiency reasons (Hidalgo et al., 2009) even if the scroll coils seems to be competitive (Grant et al., 2010; Mem et al., 2013). The use of ribbon wire instead of round wire is guided by homogeneity considerations over the sample volume.

The homogeneity of the image remains however an important issue for all MRI experiments. MRI coil designers usually anticipate it through a magnetic field intensity mapping (Mispelter et al., 2006; Hidalgo et al., 2009; Mem et al., 2013). Neglecting the MRI pulse sequence dependency a contrario, some authors have deduced the mapping of the radio-frequency coil using sequence dependency (Akoka et al., 1993; Insko and Bolinger, 1993) to correct it a posteriori. The purpose of this work is to derive a simple analytical model of the induced voltage for the solenoid coil used in emitting-receiving mode, which anticipates the effect of the MRI pulse sequence. We believe this analytic model could offer a useful tool to guide the MRI coil designer by evaluating the signal homogeneity in the longitudinal direction of the solenoid prior to its realization. The method could be applied to other coils and combined with magnetic field numerical simulation, to get it in the whole sample volume.

\section{The nuclear magnetic resonance (NMR)-induced voltage: from global to local}

When the magnetic field $\left(B_{0}\right)$ is applied to paramagnetic matter a macroscopic nuclear moment $\left(M_{0}\right)$ arises while precessing at the Larmor frequency $\omega_{0}$ (Bloch, 1946):

$\omega_{0}=-\gamma B_{0}$.

The intensity of the net magnetic moment depends on the intensity of the "polarization" magnetic field $B_{0}$ (assumed in $z$ direction following Fig. 3). Then, a varying magnetic field at Larmor frequency $\left(B_{1}\right)$ is used to rotate the magnetization transverse to the polarizing magnetic field (cf. Fig. 4). The flip angle of the magnetization $(\theta)$ will depend on $B_{1}$ magnitude and duration $(\tau)$ :

$\theta=-\gamma B_{1} \tau$

After application of $B_{1}$ magnetic field, the spins precess transversally to $B_{0}$ and are associated with an electromagnetic field whose magnetic component is classically measured by means of a coil.

The pioneer work on NMR antenna from Hoult and Richards (1976) invokes the Lorentz's reciprocity theorem to give a formulation of the induction law suited to NMR experiments:

$\xi=-\int_{\text {Sample }} \frac{\partial\left(\frac{\boldsymbol{B}_{1}}{I} \boldsymbol{M}\right)}{\partial t} \mathrm{~d} V_{\mathrm{S}}$,

where $\xi$ is the electromotive force, $\boldsymbol{B}_{1}$ is the varying magnetic field, $I$ is the electrical current, $\boldsymbol{M}$ is the magnetization of the sample and $V_{\mathrm{S}}$ is the sample volume. This formula is a well-known basis for the NMR coil SNR formulation. SNR is one of the most important parameter featuring the antenna efficiency, the other one being the homogeneity of the radio-frequency magnetic field over the sample. However, the equation derived by Hoult and Richard (namely Eq. 3) hides the dependency of the detected signal to the location of the spins while it is the quintessence of NMRI-induced voltage.

For this reason, the formulation of the induced voltage due to local elementary magnetization proposed by Pimmel (1990) in his PhD work (which is unfortunately in French but has been reported in the book of Mispelter et al., 2006) is a well suited approach to describe the NMRI signal dependency on the magnetization location $\boldsymbol{r}=(x, y, z)$ :

$\delta e(t)=-\frac{\partial}{\partial t}\left(\delta \boldsymbol{m}(t) \cdot\left(\boldsymbol{B}_{1}(\boldsymbol{r}) / I\right)\right)$ 
where $\delta e(t)$ is the contribution to the total electromotive force of the elementary magnetization $\delta \boldsymbol{m}(t)$ when a magnetic field by unit current $\boldsymbol{B}_{1}(x, y, z)$ is applied also at point $(x, y, z)$. This formulation assumes the elementary magnetization at point $(x, y, z)$ is constant (equal to $M_{0} \delta V_{\mathrm{e}}$, where $\delta V_{\mathrm{e}}$ is the volume element), but the effect of the pulse sequence on the local magnetization is not implicit. This 3-D formulation of the local contribution is elegant but confusing since the precessing component of the magnetization only appears in the plane perpendicular to $B_{0}$. For this reason, the local contribution to the induced voltage of the NMR signal proposed by Jacquinot and Sakellariou (2011) gives a more precise indication of the problem:

$\delta e(\boldsymbol{r})=-\frac{\partial}{\partial t}\left[\delta \boldsymbol{m}_{\perp}(\boldsymbol{r}) \cdot\left(\boldsymbol{B}_{1 \perp}(\boldsymbol{r}) / I\right)\right]$,

where $\boldsymbol{B}_{1 \perp}(\boldsymbol{r})$ and $\delta \boldsymbol{m}_{\perp}(\boldsymbol{r})$ are the components in the plane perpendicular to $\boldsymbol{B}_{0}$.

A generalization of the induced voltage, taking into account the propagative phenomena in the sample, is proposed by Insko et al. (1998). Thus Eq. (3) is generalized to

$\delta e(\boldsymbol{r})=-\frac{\partial}{\partial t}\left[\delta \boldsymbol{m}_{\perp}(\boldsymbol{r}) \cdot\left(\boldsymbol{B}^{\prime}{ }_{1 \perp}(\boldsymbol{r}) / I\right)\right]$,

where $\boldsymbol{B}^{\prime}{ }_{1 \perp}(\boldsymbol{r})$ is the generalized magnetic field retarded potential form:

$\boldsymbol{B}^{\prime}{ }_{1 \perp}(\boldsymbol{r})=\frac{\mu_{0}}{4 \pi} \oint e^{\mathrm{ikr}}(1-\mathrm{ikr}) \frac{\mathrm{d} \boldsymbol{l} \times \boldsymbol{r}}{\|\boldsymbol{r}\|^{3}}$,

where $k=\omega \sqrt{\varepsilon_{\mathrm{r}} \varepsilon_{0} \mu_{\mathrm{r}} \mu_{0}}$ is the wave number, $\varepsilon_{\mathrm{r}}$ is the relative permittivity of the sample and $\mu_{\mathrm{r}}$ its relative permeability. As emphasized by Insko et al. (1998), the $e^{\mathrm{ikr}}(1-\mathrm{ikr})$ term can be omitted in the near-field approximation (for $\mathrm{kr} \ll 1$ it follows $\left.e^{\mathrm{ikr}}(1-\mathrm{ikr}) \rightarrow 1\right)$.

When we try to feel the nature of the induced voltage by means of Eq. (3), we have to face some inconsistencies. First, at the time where the induced voltage is measured the magnetic field $B_{1}$ and the radio-frequency current $I$ are both null, and consequently the term $B_{1} / I$ is undefined. Second, the scalar product between the magnetization vector and a magnetic field is usually associated with the Zeeman energy, which is confusing. So, even if it is remarkably true from a mathematical point of view, the magnificent intuition of Hoult and Richard, which have gave birth to their famous formula, leads to misunderstanding for beginners. For these reasons, we derive below another way to write the NMRIinduced voltage.

We start from the mathematical form of the vector potential $(\boldsymbol{A})$ associated with the magnetic dipole moment $(\delta \boldsymbol{m})$ corresponding to the magnetization of a small volume $(\delta \boldsymbol{m}=$ $\left.M_{0} \delta V\right)$ :

$\boldsymbol{A}=\frac{\mu_{0}}{4 \pi} \frac{\delta \boldsymbol{m} \times \boldsymbol{r}}{\|\boldsymbol{r}\|^{3}} f(r)$,

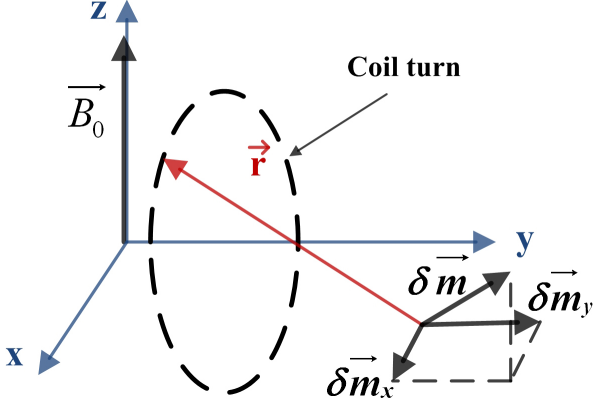

Figure 2. Illustration of the local elementary magnetization $(\delta \boldsymbol{m})$ position with respect to the coil turn for the vector potential calculation over the turn.

where $\boldsymbol{r}$ is the distance vector from the elementary magnetization location to the point where the vector potential is computed (cf. Fig. 2) and $f(r)=e^{\mathrm{ikr}}(1-\mathrm{ikr})$ summarizes the contribution of the near and far field (Insko et al., 1998).

Next, from the relation between the magnetic field $(\boldsymbol{B})$ and the vector potential $(\boldsymbol{A})$,

$\boldsymbol{B}=\bigvee \times \boldsymbol{A}$.

From Stokes theorem, for the magnetic flux through the surface, $(S)$

$\delta \phi=\iint_{(S)} \boldsymbol{B} \mathrm{d} \boldsymbol{S}=\iint_{(S)} \nabla \times \boldsymbol{A} \mathrm{d} \boldsymbol{S}=\oint \boldsymbol{A} \mathrm{d} \boldsymbol{l}$.

By substituting the vector potential by its mathematical equation (as given by Eq. 8),

$\delta \phi=\oint \frac{\mu_{0}}{4 \pi} \frac{\delta \boldsymbol{m}(\boldsymbol{r}, t) \times \boldsymbol{r}}{\|\boldsymbol{r}\|^{3}} f(r) \mathrm{d} \boldsymbol{l}$,

the total flux $\Phi$, which represents the summation over $N$ turns, is

$\Phi=N \oint \frac{\mu_{0}}{4 \pi} \frac{\delta \boldsymbol{m}(\boldsymbol{r}, t) \times \boldsymbol{r}}{\|\boldsymbol{r}\|^{3}} f(r) \mathrm{d} \boldsymbol{l}$,

which allows one to derive the local magnetization contribution to the induced voltage:

$\delta e(\boldsymbol{r}, t)=-N \frac{\partial}{\partial t} \oint \frac{\mu_{0}}{4 \pi} \frac{\delta \boldsymbol{m}(\boldsymbol{r}, t) \times \boldsymbol{r}}{\|\boldsymbol{r}\|^{3}} f(r) \mathrm{d} \boldsymbol{l}$.

By decomposing the components as $\delta \boldsymbol{m}=\delta m_{x} \boldsymbol{x}+\delta m_{y} \boldsymbol{y}$, we can write

$$
\begin{aligned}
\delta e(\boldsymbol{r}, t)= & -\frac{N \mu_{0}}{4 \pi} \oint f(r) \frac{\boldsymbol{x} \times \boldsymbol{r}}{\|\boldsymbol{r}\|^{3}} \mathrm{~d} \boldsymbol{l} \frac{\partial \delta m_{x}(\boldsymbol{r}, t)}{\partial t} \\
& -\frac{N \mu_{0}}{4 \pi} \oint f(r) \frac{\boldsymbol{y} \times \boldsymbol{r}}{\|\boldsymbol{r}\|^{3}} \mathrm{~d} \boldsymbol{l} \frac{\partial \delta m_{y}(\boldsymbol{r}, t)}{\partial t} .
\end{aligned}
$$

Finally, we can write the local magnetization contribution to the induced voltage in the standard way of writing a signal 
coming from a sensor which involves the physical quantity to be measured (here the magnetization) multiplied by the sensor sensitivity (a coefficient or a function $S$ ):

$\delta e(\boldsymbol{r}, t)=-\left[S_{x}(\boldsymbol{r}) \frac{\partial \delta m_{x}(\boldsymbol{r}, t)}{\partial t}+S_{y}(\boldsymbol{r}) \frac{\partial \delta m_{y}(\boldsymbol{r}, t)}{\partial t}\right]$,

where $\delta e(\boldsymbol{r}, t)$ represents the induced voltage, $\delta m_{x}(\boldsymbol{r}, t)$ and $\delta m_{y}(\boldsymbol{r}, t)$ are respectively the $x$ and $y$ component of the magnetization vector $\delta \boldsymbol{m}$, while $S_{x}(\boldsymbol{r})$ and $S_{y}(\boldsymbol{r})$ are the local coil sensitivities in $x$ and $y$ directions related to the coil geometry, defined as

$S_{x}(\boldsymbol{r})=\frac{\mu_{0} N}{4 \pi} \oint f(r) \frac{\boldsymbol{x} \times \boldsymbol{r}}{\|\boldsymbol{r}\|^{3}} \mathrm{~d} \boldsymbol{l}$,

$S_{y}(\boldsymbol{r})=\frac{\mu_{0} N}{4 \pi} \oint f(r) \frac{\boldsymbol{y} \times \boldsymbol{r}}{\|\boldsymbol{r}\|^{3}} \mathrm{~d} \boldsymbol{l}$,

where $\boldsymbol{x}$ and $\boldsymbol{y}$ are the unit vectors along $x$ and $y$ axis.

By virtue of the scalar triple product, the sensor's sensitivity coefficient can also be expressed:

$S_{x}(\boldsymbol{r})=-\frac{\mu_{0} N}{4 \pi} \boldsymbol{x} \cdot \oint f(r) \frac{\mathrm{d} \boldsymbol{l} \times \boldsymbol{r}}{\|\boldsymbol{r}\|^{3}}$,

$S_{y}(\boldsymbol{r})=-\frac{\mu_{0} N}{4 \pi} \boldsymbol{y} \cdot \oint f(r) \frac{\mathrm{d} \boldsymbol{l} \times \boldsymbol{r}}{\|\boldsymbol{r}\|^{3}}$,

where we definitively recognize the Biot-Savart law at a sign nearby (or the classical $\boldsymbol{B} / I$ term multiplied by the units vector). The sign difference comes from the reverse $\boldsymbol{r}$ direction convention between the usual form of Biot and Savart law with respect to the vector potential writing of Eq. (8) (Insko et al., 1998).

\section{1-D NMRI signal modeling of a ribbon solenoid coil}

In this section, we will detail the calculation of the induced voltage. We will perform the calculation on $N$ turns of ribbon solenoid of length $L$ and radius $R$ as the one represented in Fig. 3. The helicity of the antenna will be neglected. We assume a perfect homogeneity in the transverse plane $(x-z)$, which is a valid hypothesis for solenoid where the sample is not too close to the coil's wire, as reported in Hidalgo et al. (2009). Next, we assume a homogeneous current distribution flowing through the conductor. Moreover the time propagative phenomenon in the coil can be neglected since we will assume that total wire length will remain much smaller than $\lambda / 2$. Then, as discussed in Hoult (2009), we can neglect the far-field contribution (and consequently $f(r)$ is assumed close to 1) even if, according to Insko et al. (1998), it seems to be a rough assumption since $\mathrm{kr}$ value at $400 \mathrm{MHz}$ is close to 1 . Lastly, the elementary magnetization will be designated as $M$ in the following for simplicity's sake.

The NMRI coil is supposed to be used both in emitter and receiver mode. We will discuss in the following how the magnetization is tilted when the coil is used in emitter mode and

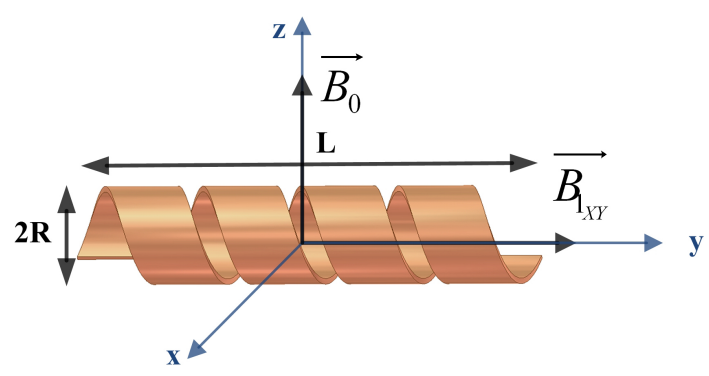

Figure 3. Illustration of the ribbon solenoid coil.

how the signal is detected by the coil when it is used in receiver mode.

\subsection{Emitter mode: the magnetization tilt}

The magnetic field component generated by a solenoid coil (Fig. 3) on $y$ axis $\left(B_{1}(y, t)\right)$ is given by Biot and Savart's law. This one can be formulated using sensitivity equations:

$B_{1}(y, t)=-S_{y}(y) I(t)$,

where $S_{y}(y)$ is well known for a solenoid while it could be calculated using Eq. (19). It follows that

$S_{y}(y)=$

$-\frac{\mu_{0} N}{2 L}\left|\frac{L / 2+y}{\sqrt{R^{2}+(L / 2+y)^{2}}}+\frac{L / 2-y}{\sqrt{R^{2}+(L / 2-y)^{2}}}\right|$.

In the following, the time dependence of both $B_{1}$ and $I$ will be omitted.

Next, by expanding Eq. (2), the distribution of the angle magnetization along the $y$ axis $(\theta(y))$ will be directly related to the magnetic field distribution:

$\theta(y)=\gamma B_{1}(y) \tau$.

Practically, the tilt angle magnetization distribution will be related to the calibration pulse sequence conditions. In this study we assume a calibration pulse performed on a small thickness slice at the center of the antenna (i.e., $y=0$ ). In case of a different pulse condition (for instance $\pi / 2$ pulse obtained over the whole sample volume) the modeling of the magnetization tilt would differ.

Thus, under the hypothesis of a centered pulse calibration, the $\pi / 2$ magnetization angle is expressed:

$$
\frac{\pi}{2}=\gamma B_{1}(0) \tau_{0},
$$

where $B_{1}(0)$ is the magnetic field at the center of the antenna and $\tau_{0}$ is the pulse duration.

By combining Eqs. (22) and (23), it appears that tilt angle is proportional to magnetic field $\left(B_{1}(y)\right)$ independently to the 


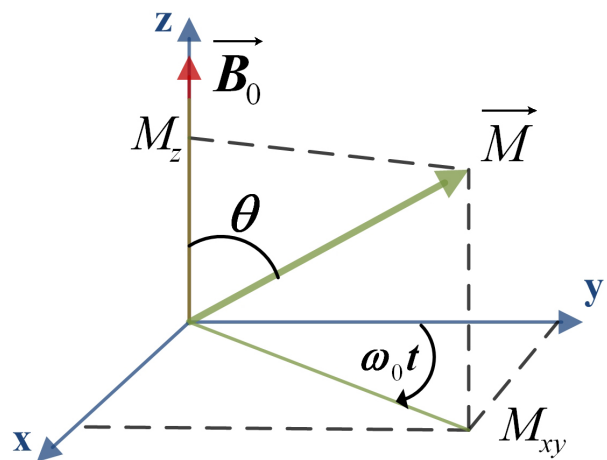

Figure 4. Magnetization vector representation in the static frame.

hardware strategy (adjustment of magnetic field magnitude or pulse duration):

$\theta(y)=\left(\frac{\pi}{2} \frac{\tau}{B_{1}(0) \tau_{0}}\right) B_{1}(y)$.

Finally, the components of the magnetization vector $\boldsymbol{M}=$ $\left[M_{x} ; M_{y} ; M_{z}\right]$ represented in Fig. 4 are expressed:

$$
\left(\begin{array}{c}
M_{x y}(y) \sin \left(\omega_{0} t\right) \\
M_{x y}(y) \cos \left(\omega_{0} t\right) \\
M_{z}(y)
\end{array}\right)
$$

where $M_{x y}(y)$ is the magnetization magnitude in the $x-y$ plane.

\subsubsection{Single pulse sequence: the magnetization tilt}

In the context of NMR single pulse sequence, the magnetization components will take the following form:

$$
\left(\begin{array}{c}
M_{0} \sin (\theta(y)) e^{-\frac{T_{\mathrm{E}}}{T_{2}^{*}}}\left(1-e^{-\frac{T_{\mathrm{R}}}{T_{1}}}\right) \sin \left(\omega_{0} t\right) \\
M_{0} \sin (\theta(y)) e^{-\frac{T_{\mathrm{E}}}{T_{2}^{*}}}\left(1-e^{-\frac{T_{\mathrm{R}}}{T_{1}}}\right) \cos \left(\omega_{0} t\right) \\
M_{0} \cos (\theta(y))\left(1-e^{\frac{T_{\mathrm{R}}}{T_{1}}}\right)
\end{array}\right),
$$

where $\left(T_{1}\right)$ is the longitudinal relaxation time, $\left(T_{\mathrm{R}}\right)$ is the repetition time, $\left(T_{2}^{*}\right)$ is the transverse relaxation time in a heterogeneous magnetic field and $\left(T_{\mathrm{E}}\right)$ is the echo time.

Let us now determine the flip angle distribution for two important MRI pulse sequences, namely gradient echo and spin echo.

\subsubsection{Gradient echo sequence: the magnetization tilt}

For a gradient echo sequence it is a desirable condition to perform it at the Ernst angle ( $\left.\theta_{\text {ERNST }}\right)$. Following the same reasoning that has led to Eq. (24), the flip angle distribution $(\theta(y))$ for a gradient echo sequence will follow

$\theta(y)=\theta_{\text {ERNST }} \frac{B_{1}(y) \tau}{B_{1}(0) \tau_{\text {ERNST }}}$, where $\tau_{\text {ERNST }}$ is the pulse duration required to tilt the magnetization at Ernst angle at the center of the sample.

Thus, the magnetization in $x-y$ plane (component $M_{x y}(y)$, cf. Eq. 25) will be

$M_{x y}(y)=M_{0} \frac{\sin (\theta(y))\left(1-e^{-\frac{T_{\mathrm{R}}}{T_{1}}}\right)}{1-\cos (\theta(y)) e^{-\frac{T_{\mathrm{R}}}{T_{1}}}} e^{-\frac{T_{\mathrm{E}}}{T_{2}^{*}}}$,

where $\theta(y)$ is the magnetization angle distribution given by Eq. (27).

\subsubsection{Spin echo sequence: the magnetization tilt}

For the spin echo sequence, the magnetization will be tilted by a $\pi / 2$ pulse followed by a $\pi$ pulse. We use the signal dependency given by Akoka et al. (1993) and Insko and Bolinger (1993) for spin echo sequence: $\sin ^{3}(\theta)$. Thus the magnetization in $x-y$ plane $\left(M_{x y}(\theta(y))\right)$ will be given by

$M_{x y}(\theta(y))=M_{0} \sin ^{3}(\theta(y))\left(1-e^{-\frac{T_{\mathrm{R}}}{T_{1}}}\right) e^{-\frac{T_{\mathrm{E}}}{T_{2}}}$,

where $\theta(y)$ is the magnetization angle distribution given by Eq. (24) and $\left(T_{2}\right)$ is the true transverse relaxation time.

\subsection{Receiver mode: the induced voltage}

Once the magnetization flip is determined, we can establish the induced voltage associated with the magnetization precession. The induced voltage created by the elementary magnetization at location $y$ can be simply expressed from Eq. (15) by considering only the sensitivity along $y$ axis:

$e(t, y)=-S_{y}(y) \frac{\mathrm{d} M_{y}(y)}{\mathrm{d} t}$,

where $S_{y}(y)$ is deduced from Eq. (21).

Since $M_{y}(y)=M_{x y}(y) \cos \left(\omega_{0} t\right)$, the induced voltage in harmonic regime will be

$|\underline{e}(y)|=\left|\omega_{0} S_{y}(y) M_{x y}(y)\right|$,

where $M_{x y}(\theta(y))$ is given either by Eqs. (28) or (29) depending of the running pulse sequence.

\section{Design of the NMRI ribbon solenoid coil}

To design the NMRI ribbon solenoid coil, the first point is to determine the total length of the wire $\left(L_{\mathrm{w}}\right)$. In order to neglect the propagative phenomenon into the coil, a length of the solenoid coil about $\approx \lambda / 6$ is classically used (Mispelter et al., 2006), while $\lambda$ is determined by the nuclear frequency of interest into the magnet. In our study, we performed $1 \mathrm{H}$ measurement on a 9.4 T Varian MRI. Thus, the gyromagnetic 
Table 1. Solenoid coil design parameter summary.

\begin{tabular}{llll}
\hline$N$ & $D(\mathrm{~mm})$ & $w(\mathrm{~mm})$ & $t(\mathrm{~mm})$ \\
\hline 3 & 8 & 10 & 2 \\
\hline
\end{tabular}

frequency $\left(f_{0}\right)$ is about $400 \mathrm{MHz}$, and it follows that the total length of the solenoid coil should be limited to $12.5 \mathrm{~cm}$ :

$L_{\mathrm{w}}=\frac{\pi N D}{\cos (\psi)} \leq \lambda / 6$

where $\psi$ is the pitch angle. The turn number is then deduced from the size of the sample (or its mechanical support). In our study the tissue length is $\sim 40 \mathrm{~mm}$ while the tube diameter $(D)$ is equal to $8 \mathrm{~mm}$. The copper ribbon has $10 \mathrm{~mm}$ width $(w)$ and $50 \mu \mathrm{m}$ thickness $\left(t_{\mathrm{w}}\right)$. From Eq. (32) it follows that $N=3$. Choosing a space between turns $(t)$ equal to $2 \mathrm{~mm}$ results in an average length of the solenoid $L \sim 36 \mathrm{~mm}$ (the design parameters are summarized in Table 1).

\subsection{NMRI coil electrical model}

Basically, the coil can be represented by the electromotive force (given by Eq. 15) in series with an inductance ( $L 1)$ and a resistance which takes into account the occurrence of the skin effect $\left(R 1_{\mathrm{AC}}\right)$. When considering a single ribbon, the current density will tend to flow at the ends of the ribbon: this effect is known as lateral skin effect (Belevitch, 1971). When considering a multiple-turn solenoid (ribbon or round wire), the current density between neighbor conductors will be strengthened especially at the extremities of the coil: this effect is known as the proximity skin effect (Butterworth, 1925). The analytic modeling of these phenomena is beyond the scope of this paper, but they can be efficiently approached by electromagnetic numerical simulations. Finally these effects will dictate the current distribution at high frequencies and thus the homogeneity. In practice, even if it increases the coil's resistance and thus the noise, the use of ribbon wire tends to improve the homogeneity (Grant et al., 2010; Mem et al., 2013). In the case of ribbon solenoid the spacing between turns should be minimized in order to preserve homogeneity on one side but should be sufficient to avoid to strengthen the proximity effect on the other side. The different skins effects are illustrated in Fig. 5.

Lastly, the occurrence of the coil's resonance at a frequency $\left(f_{0}\right)$ where the wavelength $(\lambda)$ is about twice the wire length (namely $\lambda=c / f_{0} \simeq 2 L_{c}$ where $c$ is the vacuum light velocity), will imply $\left.f_{0} \simeq c /\left(2 L_{c}\right)\right)$ can be interpreted by a capacitance $(C 1)$ in parallel (cf. Fig. 6) with the previous components (Knight, 2013b).

\subsection{Tuning-matching circuit}

The intrinsic self-resonance of the coil is much higher than the one to observe; moreover the electrical resonance fre- (a)

(b)

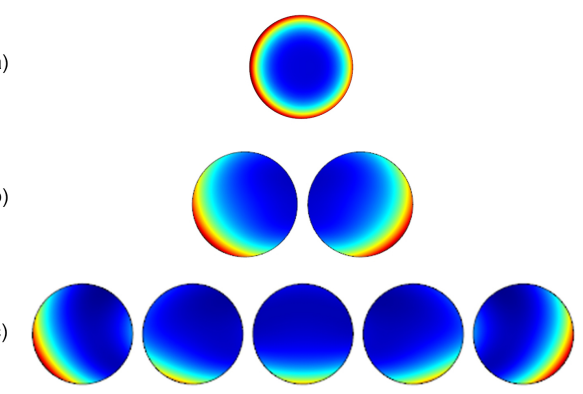

Figure 5. Illustration of the different skin effect regimes in the section of one, two and five turns: (a) usual skin effect, (b) proximity skin effect and (c) lateral skin effect.

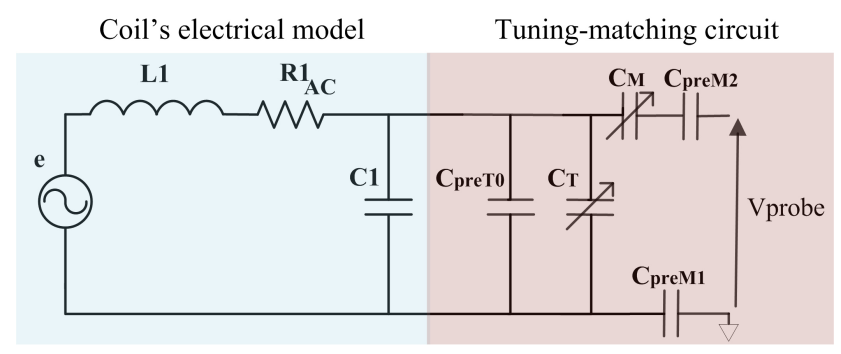

Figure 6. Electrical circuit of the coil plus the tuning-matching circuit.

quency will be affected by the sample dielectric properties. For these reasons, a variable capacitance is usually added in parallel to the coil terminal to adjust the resonance frequency (the tuning circuit is represented by capacitances $C_{\mathrm{T}}$ and $C_{\text {preT }}$ in Fig. 6). On the other side, the coil must be connected to the radio-frequency power amplifier and impedance of the coil must be matched to the standard $50 \Omega$ at the frequency of use. For this purpose the capacitances $C_{\mathrm{M}}, C_{\mathrm{preM}} 1$ and $C_{\mathrm{preM} 2}$ are used in series (the electrical component values are summarized in Table 2).

\section{Experimental results}

The experiments have been performed on a $9.4 \mathrm{~T}$ magnet from AGILENT. The ribbon solenoid coil has been wound on a glass tube. A small printed circuit board (PCB) is used to realize the tuning-matching circuit. Variable non-magnetic capacitances from VOLTRONICS (Ref. NMKJ10HVE from 0.5 up to $9 \mathrm{pF}$ ) are used. Copper foil connected to the ground BNC cable has been used on the reverse face of the mechanical structure to perform an electromagnetic shielding and to prevent the sensibility of the circuit during the manual adjustment. Special care has been given to the connection distance between the ground and the copper foil to prevent occurrence of resonance in the frequency range of interest. A mechanical structure to maintain the glass tube and the PCB, represented in Fig. 7, has been realized using a 3-D printer with polylactic acid material. 
Table 2. Electrical parameter summary.

\begin{tabular}{lllllll}
\hline$R 1_{\mathrm{AC}}$ & $L 1$ & $C 1(\mathrm{pF})$ & $C_{\text {preT0 }}$ & $C_{\text {preM1 }}$ & $C_{\text {preM } 2}$ & $Q$ \\
\hline$\sim 0.3(\Omega)$ & $15 \mathrm{nH}$ & $0.55 \mathrm{pF}$ & $6.8 \mathrm{pF}$ & $0.4 \mathrm{pF}$ & $0.4 \mathrm{pF}$ & 135 \\
\hline
\end{tabular}

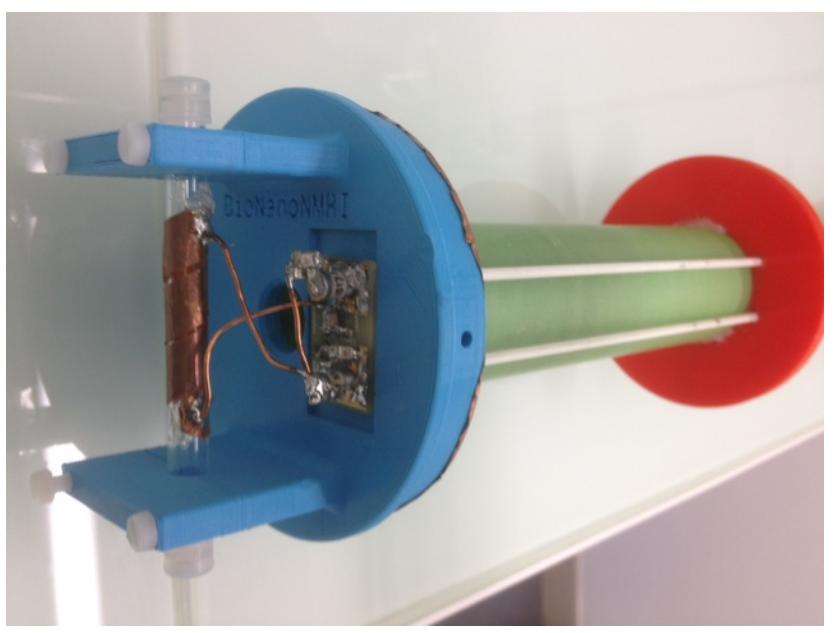

Figure 7. Photograph of the ribbon solenoid coil dedicated to spinal cord injury.

The pulse power needed to tilt the magnetization at $\pi / 2$ (on a $100 \mathrm{~mm}$ length and $4 \mathrm{~mm}$ diameter glass tube filled with potable water) in a centered thin slice is about $4.8 \mathrm{~dB}$ (the SNR is $1600 / 45$, obtained using a gradient echo sequence with the following parameters: $\mathrm{FOV}=10 \times 10: \mathrm{TR}=250 \mathrm{~ms}$, $\mathrm{TE}=4.32 \mathrm{~ms}$, flip angle $=80^{\circ}$, average $=2$, resolution $128 \times$ 128,20 slices of thickness $=1 \mathrm{~mm}$ ). For comparison, the pulse power, on the same water sample, needed by a commercial volumetric quadrature antenna with $43 \mathrm{~mm}$ inner diameter (from RAPID Biomedical) in the same conditions is about $20 \mathrm{~dB}$ (the SNR on the image in the same conditions is 290/45). The enhancement of the SNR between the ribbon solenoid coil and the commercial antenna is $\sim 5.5$, which is well correlated with the pulse power attenuation. This increase of the SNR allows one either to perform faster acquisition ( $\sim 25$ times) for a given resolution or to enhance the resolution for a given acquisition time. In the context of spinal cord injury studies, this improvement in the SNR (as demonstrated on a T2-weighted spin echo sequence in Fig. 8) was crucial. The time acquisition has been divided by 10 while the image quality has been significantly enhanced allowing one to combine high-resolution T2-weighted acquisition and diffusion MRI imaging to investigate accurately the lesion site of the spinal cord.

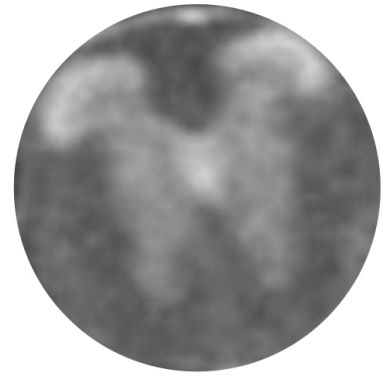

(a)

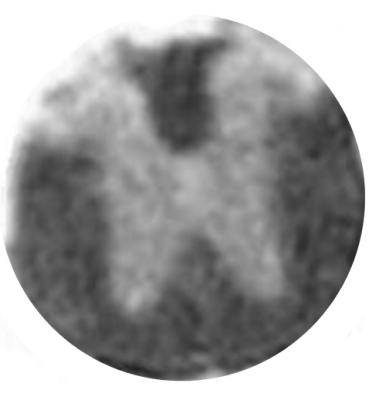

(b)
Figure 8. Ex vivo MR images (multi-echo multi-slice sequence: $\mathrm{TR}=1155 \mathrm{~ms} ; \quad \mathrm{TE}=14 \mathrm{~ms} ; \quad \mathrm{NE}=1 ; \quad \mathrm{FOV}=10 \mathrm{~mm} \times 10 \mathrm{~mm} ;$ 60 slices; thickness $=0.6 \mathrm{~mm}$; resolution $=256 \times 256$ ) of spinal cords from adult mice. Hypersignal (butterfly shape) represents the grey matter whereas the surrounding hyposignal corresponds to the white matter: (a) image obtained with RAPID Biomedical $43 \mathrm{~mm}$ volumic quadrature coil in $14 \mathrm{~h} 0 \mathrm{~min}$; (b) image obtained with the ribbon solenoid coil of Fig. 7 in $1 \mathrm{~h} 30 \mathrm{~min}$.

\subsection{Gradient echo sequence: method and experimental results}

According to Eq. (27), $\theta$ is proportional to $B_{1}(y) / y$, which is equivalent to the sensitivity term $S_{y}(y)$, resulting in $\theta(y) \propto$ $S_{y}(y)$. Then, we normalize the tilt angle distribution to the Ernst angle:

$\theta(y)=\theta_{\operatorname{ERNST}}(y) \frac{S_{y}(y)}{S_{y}(0)}$,

where $S_{y}(y)$ is given by Eq. (21). Combining Eqs. (28) and (33) into the MRI-induced voltage (Eq. 31) leads to

$$
e(y) \propto \theta(y) \frac{\sin (\theta(y))}{\left(1-\cos (\theta(y)) e^{-\frac{T_{\mathrm{R}}}{T_{1}}}\right.} e^{-\frac{T_{\mathrm{E}}}{T_{2}^{*}}} .
$$

For a given set of experimental conditions parameters $\left(T_{\mathrm{R}}\right.$, $\left.T_{1}, \theta_{\text {ERNST }}\right)$ and given solenoid coil size parameters, the induced voltage $e(y)$ can be plotted. The model (values are normalized) is compared to the normalized experimental data (cf. Fig. 10).

\subsection{Spin echo sequence: method and experimental results}

For a spin echo sequence, the distribution of the magnetization angle tilt will be

$\theta(y)=\frac{\pi}{2} \frac{S_{y}(y)}{S_{y}(0)}$, 


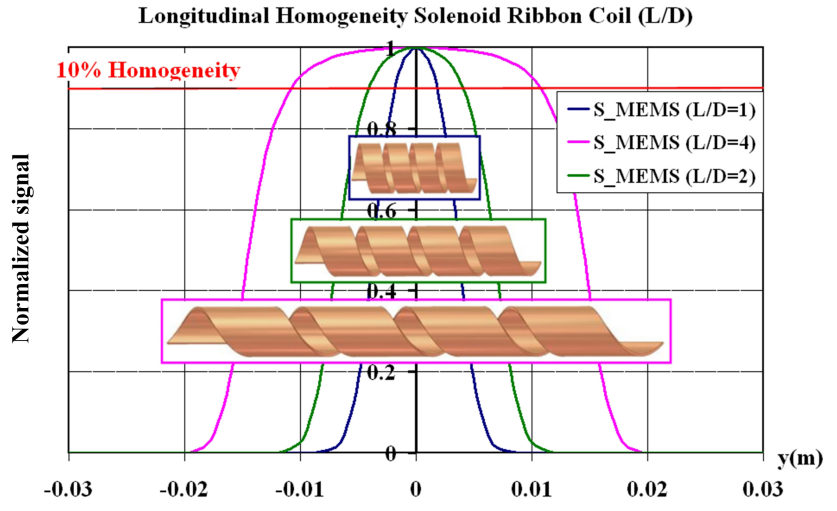

Figure 9. Comparisons of longitudinal homogeneity profiles for different solenoid coil aspect ratios.

where $S_{y}(y)$ is given by Eq. (21). Combining Eqs. (29) and (35) into the MRI-induced voltage (Eq. 31) leads to

$e(y) \propto \theta(y) \sin ^{3}(\theta(y))\left(1-e^{-\frac{T_{\mathrm{R}}}{T_{1}}}\right) e^{-\frac{T_{\mathrm{E}}}{T_{2}}}$.

The model can be used to anticipate the longitudinal homogeneity range for different aspect ratios (i.e., $L /(2 R)$ ratios) of solenoid coils in the context of spin echo sequence. It highlights how to use the model to guide the designer. For instance, it highlights the inefficiency of the short solenoid coil where less than $50 \%$ of the length provides enough signal (considering the homogeneity criterion as $10 \%$ variation of the signal intensity).

Finally, the model so obtained is compared to experimental data in Fig. 10. The measured data have been obtained by inserting a tube of water ( $100 \mathrm{~mm}$ length) into the ribbon solenoid coil. The measurements have been done through the VNMRJ software interface using circular region of interest over the whole diameter.

The comparison between gradient echo and spin echo (Fig. 10a) allows one to illustrate the $y$ axis homogeneity dependency on the pulse sequence. The measurement also shows differences between the two type of sequences, the echo gradient sequence exhibiting a significantly wider homogeneity range. For both sequences we can notice a significant smooth decrease of the signal far from the coil in practice while the decrease is predicted as more abrupt by the model. It is certainly related to the far-field contribution predicted by Insko et al. (1998) conversely to the conclusion given by Hoult (2009).

The real ribbon solenoid coil signal exhibits some magnitude fluctuations which are attributed to the spacing between turns. The occurrence of maxima at the ends of the solenoid coil is attributed to the high-frequency current density distribution discussed above, where the different skin effects tend to distribute the current density at its ends increasing both $B_{1}$ and the sensitivity. Finally, the modeling allows the anticipation of the $y$ axis homogeneity tendency at an early stage of the coil design.
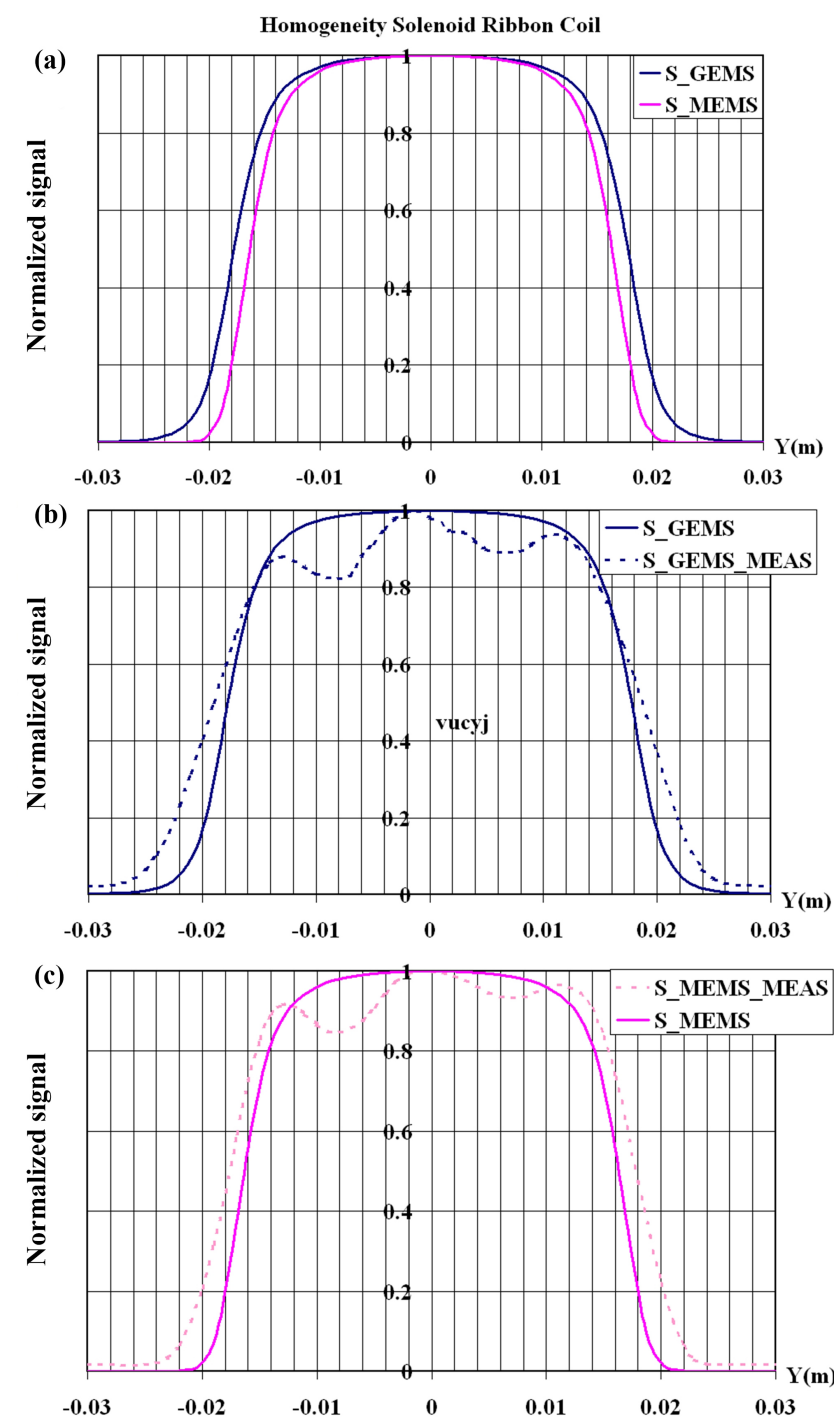

Figure 10. Normalized induced signal comparisons: (a) gradient echo sequence (GEMS) versus spin echo sequence (MEMS); (b) gradient echo sequence measured (GEMSMEAS) versus model (GEMS) $(\mathrm{TR}=688 \mathrm{~ms} ; \mathrm{TE}=4.5 \mathrm{~ms}$; FOV $=10 \mathrm{~mm} \times 10 \mathrm{~mm} ; 80$ slices; thickness $=1 \mathrm{~mm}$; resolution $=128 \times 128$ ); (c) spin echo sequence measured (MEMSMEAS) versus model (MEMS) $(\mathrm{TR}=10 \mathrm{~s} ; \quad \mathrm{TE}=10 \mathrm{~ms}$; FOV $=10 \mathrm{~mm} \times 10 \mathrm{~mm} ; 80$ slices; thickness $=1 \mathrm{~mm}$; resolution $=128 \times 128)$.

\section{Conclusions}

The step-by-step modeling presented in this paper enables the estimation of the longitudinal signal homogeneity of a solenoid coil depending on the running imaging sequence. The formulation of the local contribution of the elementary magnetization to the induced voltage, using sensitivities coefficients, derived in this paper is well suited for MRI coil designers while it avoids the use of confusing notations. We believe this type of modeling could be applied to other coil 
shapes in order to guide MRI coil designers in their choice of coils. Two important phenomena reduce the analytic modeling validity, namely, the current density distribution at high frequency and the far-field contribution, which will be more significant for higher frequencies and higher coil and sample sizes. Quantitative modeling of the homogeneity over the volume could be attained by combining classical numerical computations of magnetic field with the MRI pulse sequence conditions.

Finally, the customized antennas are a relevant and cheap way of enhancing the performance of the MRI studies with respect to the commercial antennas.

Acknowledgements. The authors would like to thank Association Verticale, who funded the 9.4 T MRI dedicated to SCI studies, and also thank the Labex NUMEV, who funded the electronic material needed to perform this study.

Edited by: I. Bársony

Reviewed by: two anonymous referees

\section{References}

Akoka, S., Franconi, F., Seguin, F., and Le Pape, A.: Radiofrequency map of an NMR coil by imaging, Magnetic Resonance Imaging, 11, 437-441, 1993.

Belevitch, V.: The lateral skin effect in a flat conductor, Philips tech Rev., 32, 221-231, 1971.

Bloch, F.: Nuclear Induction, Physical Review, 70, 460-474, 1946.

Butterworth, S.: On the alternating current resistance of solenoidal coils, Proc. R. Soc. Lon. Ser.-A, 107, 693-715, 1925.

Grant, C. V., Wu, C. H., and Opella, S. J.: Probes for high field solid-state NMR of lossy biological samples, J. Magn. Reson., 204, 180-188, 2010.

Hidalgo, S. S., Jirak, D., Solis, S. E., and Rodriguez, A. O.: Solenoid coil for mouse-model MRI with a clinical 3-Tesla imager: body imaging, Rev. Mex. Fis., 55, 140-144, 2009.
Hoult, D. I.: The Origins and Present Status of the Radio Wave Controversy in NMR, Concept. Magn. Reson. A, 34, 193-216, 2009.

Hoult, D. I. and Richards R. E.: The signal-to-noise ratio of the nuclear magnetic resonance experiment, J. Magn. Reson., 24, 7185, 1976.

Insko, E. K. and Bolinger, L.: Mapping of the Radiofrequency field, J. Magn. Reson., 103, 82-85, 1993.

Insko, E. K., Elliott, M. A., Schotland, J. C., and Leigh, J. S.: Generalized Reciprocity, J. Magn. Reson., 131, 111-117, 1998.

Jacquinot, J.-F. and Sakellariou, D.: NMR Signal Detection using Inductive Coupling: Applications to Rotating Microcoils, Concept. Magn. Reson. A, 38, 33-51, 2011.

Knight, D. W.: Solenoid Inductance Calculation, version of Sept. 2015, available at: http://www.g3ynh.info/zdocs/magnetics/ Solenoids.pdf, 2013a.

Knight, D. W.: The self-resonance and self-capacitance of solenoid coils, version of Sept. 2015, available at: http://www.g3ynh.info/, $2013 b$.

Meme, S., Joudiou, N., and Szmereta, F.: In vivo magnetic resonance microscopy of Drosophilae at 9.4T, Magn. Reson. Imaging, 31, 109-119, 2013.

Mispelter, J., Lupu, M., and Briguet, A.: NMR probehads for biophysical and biomedical experiments: theoretical principles and practical guidelines, Imperial College Press, 2006.

Noristani, H. N., Lonjon, N., Cardoso, M., Le Corre, M., ChanSeng, E., Captier, G., Privat, A., Coillot, C., Goze-Bac, C. and Perrin, F. E.: Correlation of in vivo and ex vivo 1H-MRI with histology in two severities of mouse spinal cord injury, Front. Neuroanat., 9, 24, doi:10.3389/fnana.2015.00024, 2015.

Pimmel, P.: Les Antennes en Resonance Magnétique Nucléaire: Fonctionnement et Réalisation, $\mathrm{PhD}$ Thesis, Univ. Claude Bernard, Lyon, 1990.

Sekhon, L. H. and Fehlings, M. G.: Epidemiology, demographics, and pathophysiology of acute spinal cord injury, Spine, 26, S212,2001

Webb, A. G.: Radiofrequency microcoils for magnetic resonance imaging and spectroscopy, J. Magn. Reson., 31, 55-66, 2013.

Wheeler, H. A.: Simple Inductance Formulas for Radio Coils, P. IRE, 16, 1398-1400, 1928. 